\title{
Tratamento Percutâneo de Pseudoaneurisma da Zona Fibrosa Intervalvar Mitroaórtica
}

\author{
Daniel Zanuttini', Eulogio García², Jose L. Lazarte³, Costantino O. Costantini', Sergio Tarbine', \\ Marcelo Freitas ${ }^{1}$, Marcos Denk ${ }^{1}$, Costantino R. Costantini ${ }^{1}$
}

\section{RESUMO}

O pseudoaneurisma da zona fibrosa intervalvar mitroaórtica (PFMA) é uma doença pouco frequente, em geral secundária a endocardite da valva aórtica, particularmente em próteses valvares. Seu curso clínico é variável e pode ocasionar complicações graves, como ruptura para o pericárdio, aorta ou átrio esquerdo, compressão sistólica das artérias coronárias ou compressão sistólica da valva mitral, ocasionando regurgitação mitral acentuada, motivo pelo qual se recomenda seu tratamento cirúrgico. Neste relato é apresentado o caso de paciente com 69 anos de idade, assintomático, com antecedente de duas cirurgias de revascularização miocárdica, a última associada a troca valvar aórtica. O PFMA foi diagnosticado incidentalmente na evolução pós-operatória tardia, optando-se pelo tratamento percutâneo com a prótese Amplatzer TM Muscular VSD Occluder.

DESCRITORES: Falso aneurisma. Valva aórtica. Próteses valvulares cardíacas.

A zona intervalvar mitroaórtica é uma estrutura fibrosa delgada que conecta a porção posterior da raiz aórtica à base do folheto anterior da valva mitral. Essa estrutura separa a via de saída do ventrículo esquerdo de um coxim de gordura pericárdica, presente entre a porção posterior da aorta e a parede do átrio esquerdo. ${ }^{1,2} \mathrm{O}$ pseudoaneurisma da zona fibrosa mitroaórtica (PFMA) é raro, geralmente resultante da perfuração da zona fibrosa por processo infeccioso. É caracterizado por uma cavidade pulsátil entre a aorta e o átrio esquerdo, que se comunica com o trato de saída do ventrículo esquerdo, e que pode romper,

\footnotetext{
1 Hospital Cardiológico Costantini - Curitiba, PR, Brasil.

2 Hospital General Universitario Gregorio Marañón - Madri, Espanha.

3 Instituto de Cardiologia e Cirurgia Cardiovascular de Posadas Misiones, Argentina.

Correspondência: Daniel Zanuttini. Rua Pedro Collere, 890 - Vila Izabel - Curitiba, PR, Brasil - CEP 80320-320

E-mail: danielzanuttini@hotmail.com

Recebido em: 11/4/2011 • Aceito em: 11/8/2011
}

\begin{abstract}
Percutaneous Treatment of Pseudoaneurysm of the Mitral-Aortic Intervalvular Fibrosa

Pseudoaneurysm of the mitral-aortic intervalvular fibrosa (PMAF) is a rare disease, usually secondary to aortic valve endocarditis, particularly in prosthetic valves. Its clinical course is variable and may potentially cause serious complications, such as rupture into the pericardium, aorta or left atrium, systolic compression of the coronary arteries or systolic compression of the mitral valve, leading to severe mitral regurgitation, for which surgical treatment is recommended. This is a case report of a 69 year-old asymptomatic patient, with a prior history of two coronary artery bypass graft surgeries, the latter associated with aortic valve replacement. The PMAF was incidentally diagnosed in the late follow-up, and a decision was made to perform percutaneous therapy with the Amplatzer ${ }^{\top M}$ Muscular VSD Occluder.
\end{abstract}

KEY-WORDS: Aneurysm, false. Aortic valve. Heart valve prosthesis.

mais frequentemente, para a cavidade pericárdica. A etiologia mais frequente é a endocardite da valva ou prótese aórtica. ${ }^{1,3}$ Apresentamos um caso de PFMA em paciente assintomático, com diagnóstico incidental na evolução tardia de cirurgia de troca valvar aórtica, tratado por via percutânea.

\section{RELATO DO CASO}

Paciente do sexo masculino, de 69 anos, com antecedente de cirurgia de revascularização miocárdica em 1981, em que foram colocadas pontes de safena para as artérias descendente anterior, coronária direita e circunflexa. Em 1998, foi submetido a angioplastia com implante de stent para o leito distal da ponte de safena para coronária direita; dois anos após, foi necessária nova intervenção coronária percutânea para a circunflexa e óstio da ponte de safena para artéria descendente anterior. Em 2001, em decorrência de estenose da valva aórtica, foi submetido a troca valvar por prótese biológica e nova revascularização, com enxerto arterial em "Y" para artéria descendente anterior distal 
e ramo diagonal, e ponte de safena para ramo marginal da circunflexa. Em 2005, realizou prostatectomia por neoplasia de próstata. Em 2006, submeteu-se a outra angioplastia, para tratar reestenose do stent no óstio da ponte de safena para descendente anterior.

Evoluiu assintomático, tendo realizado tomografia computadorizada de tórax para avaliação uro-oncológica em março de 2009, em que foi visualizada imagem suspeita de aneurisma coronário com protrusão para o átrio esquerdo. À angiotomografia coronária, visualizava-se imagem arredondada na união mitroaórtica, não-pulsátil, com diâmetro máximo de $51 \mathrm{~mm}$, com acentuada calcificação parietal e comunicação com trato de saída do ventrículo esquerdo através de um colo de $7 \mathrm{~mm}$ (Figura 1). A ressonância nuclear magnética confirmou os achados descritos (Figura 2). Para prevenir provável ruptura ou complicações, e pelo risco de uma terceira cirurgia cardíaca, optou-se pelo fechamento percutâneo do pseudoaneurisma.

O procedimento foi guiado por angiografia e ecocardiografia transesofágica. Por via arterial femoral direita foi posicionado cateter Amplatz (LA) $6 \mathrm{~F}$ na região ventricular esquerda, direcionado para o colo do pseudoaneurisma. Avançou-se uma guia 0,035 polegada, de $260 \mathrm{~cm}$ (Terumo Co., Tóquio, Japão), até o interior do PFMA, fazendo círculos em seu interior (Figura 3). Posteriormente trocou-se o cateter Amplatz por um cateter Multipurpose, para melhor coaxibilidade com a raiz aórtica. Trocou-se, em seguida, a guia por outra de maior suporte (Lunderquist), e posicionou-se bainha de Mullins 7 F. Através do cateter, avançou-se a prótese
Amplatzer ${ }^{\mathrm{TM}}$ Muscular VSD Occluder (AGA Medical, Golden Valley, Estados Unidos) de $12 \mathrm{~mm}$ no colo do PFMA. A posição foi controlada mediante ventriculografia em duas projeções (oblíqua anterior direita 30 graus e oblíqua anterior esquerda 45 graus) e ecocardiografia transesofágica, constatando-se que não interferia com a função da bioprótese aórtica nem com a valva mitral (Figura 4). Após sua liberação, a ecocardiografia transesofágica e a angiografia demonstraram ausência de fluxo na cavidade do PFMA (Figura 5).

Quinze meses após o fechamento do PFMA, o paciente permanece assintomático. No controle, foram realizados ecografia transtorácica, que evidenciou discreto fluxo sisto-diastólico residual periprótese de Amplatzer $^{\mathrm{TM}}$, expansão adequada da prótese no colo do PFMA, sem comprometimento da função valvar mitral nem aórtica. À angiotomografia de múltiplos cortes observou-se presença de formação sacular com parede calcificada, parcialmente trombosada, com diâmetro máximo de $51 \mathrm{~mm}$, demonstrando ausência de crescimento do PFMA em relação à avaliação pré-procedimento (Figura 6).

\section{DISCUSSÃO}

O PFMA tem sido descrito mais frequentemente associado a endocardite infecciosa da valva ou prótese aórtica.1,2 Também foram descritos casos após troca valvar aórtica, associados a deiscência parcial da prótese $^{4}$ e mesmo sem causa aparente conhecida ${ }^{5,6}$ Mais raramente têm sido descritos casos secundários a trauma torácico fechado ${ }^{7}$ ou de origem congênita. ${ }^{8}$
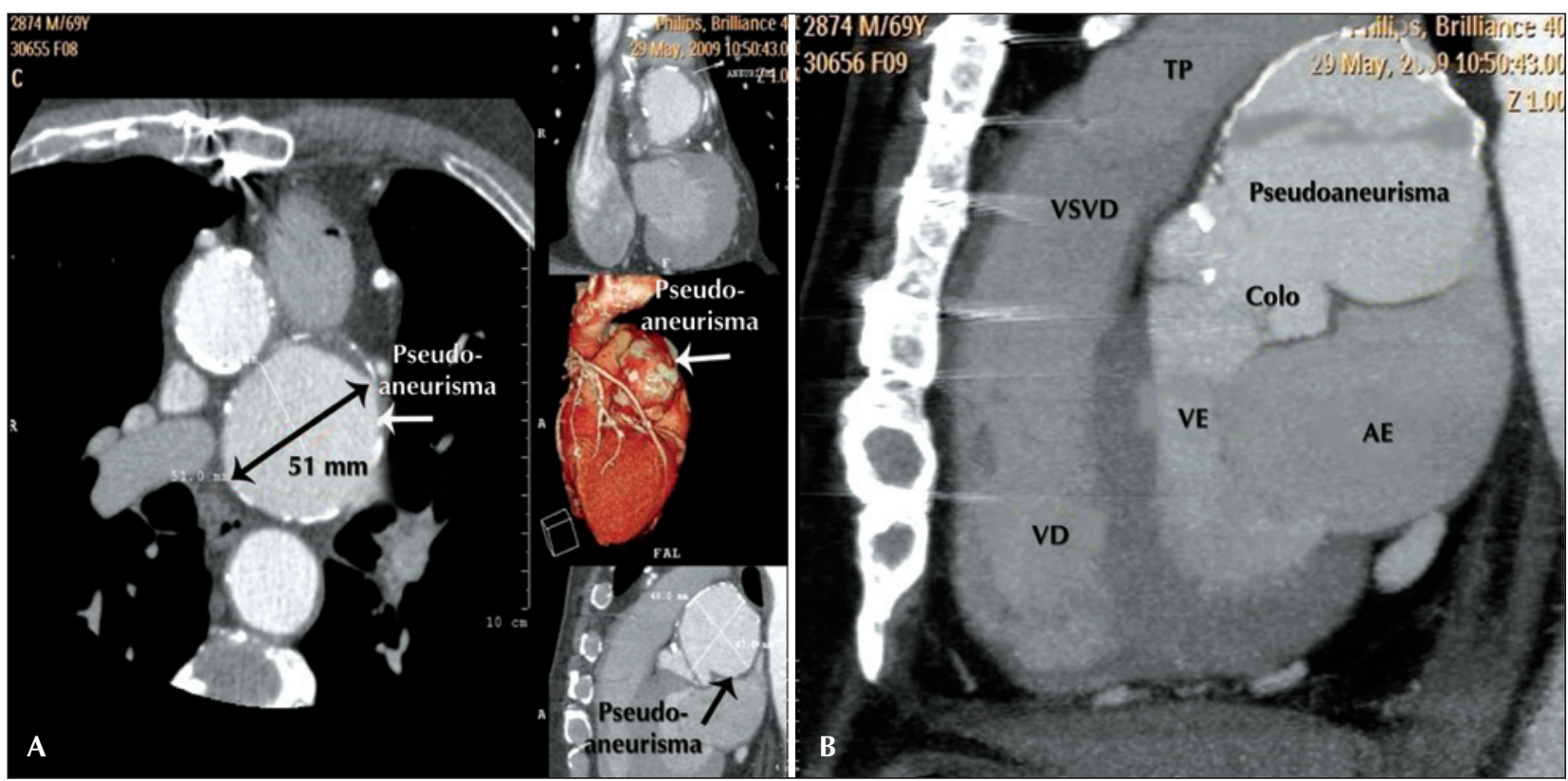

Figura 1 - Em A, tomografia de múltiplos cortes, demonstrando cavidade não-pulsátil, sacular, com diâmetro máximo de 51 mm (seta preta). Em B, comunicação do pseudoaneurisma com o trato de saída do ventrículo esquerdo, apresentando colo de 7 mm de extensão e acentuada calcificação parietal da cavidade. $\mathrm{AE}=$ átrio esquerdo; $\mathrm{TP}=$ tronco pulmonar; $\mathrm{VD}=$ ventrículo direito; $\mathrm{VE}=$ ventrículo esquerdo; $\mathrm{VSVD}=$ via de saída do ventrículo direito. 


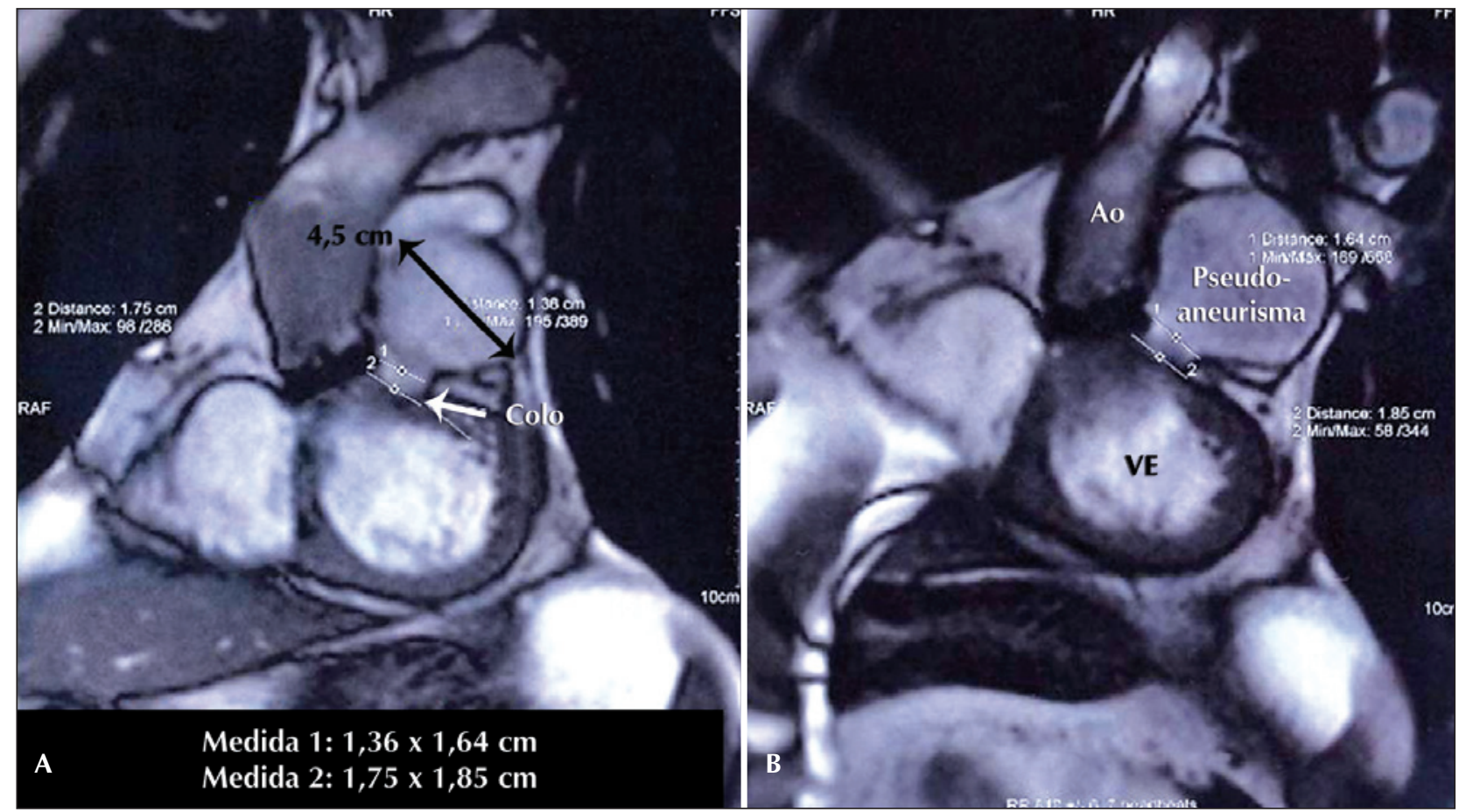

Figura 2 - Confirmação diagnóstica do pseudoaneurisma pela ressonância nuclear magnética. Ao = aorta; VE = ventrículo esquerdo.
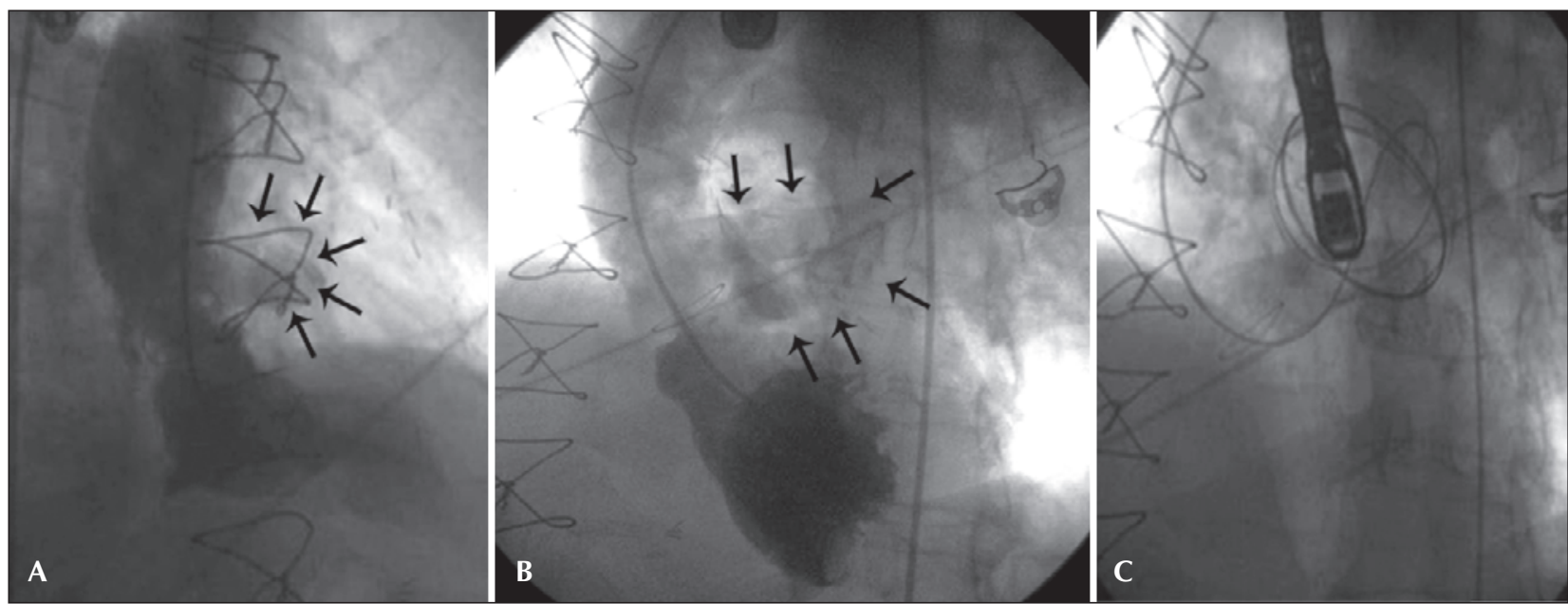

Figura 3 - Em A e B, ventriculografia em oblíqua anterior direita e esquerda, respectivamente, visualizando contraste no interior do pseudoaneurisma (setas). Em C, transdutor de ecocardiografia transesofágica e guia 0,035 polegada, posicionando no interior do pseudoaneurisma.

No caso aqui relatado o diagnóstico foi feito na fase pós-operatória tardia de duas cirurgias cardíacas, em paciente sem histórico de endocardite infecciosa diagnosticada.

O PFMA comunica-se com a via de saída do ventrículo esquerdo e, usualmente, apresenta mudanças de tamanho dinâmicas concomitantes às alterações da pressão intraventricular durante o ciclo cardíaco. A característica não-pulsátil do caso relatado o diferencia de todos os demais publicados, e a ausência de expansão sistólica e colapso diastólico possivelmente é explicada pela acentuada calcificação parietal encontrada.

A apresentação clínica é variável, podendo o paciente mostrar-se assintomático ou apresentar quadro clínico de hemopericárdio ou tamponamento cardíaco, por rotura do PFMA na cavidade pericárdica. ${ }^{9}$ Também tem sido descrita insuficiência cardíaca, pela comunicação com a aorta ascendente ou átrio esquer- 


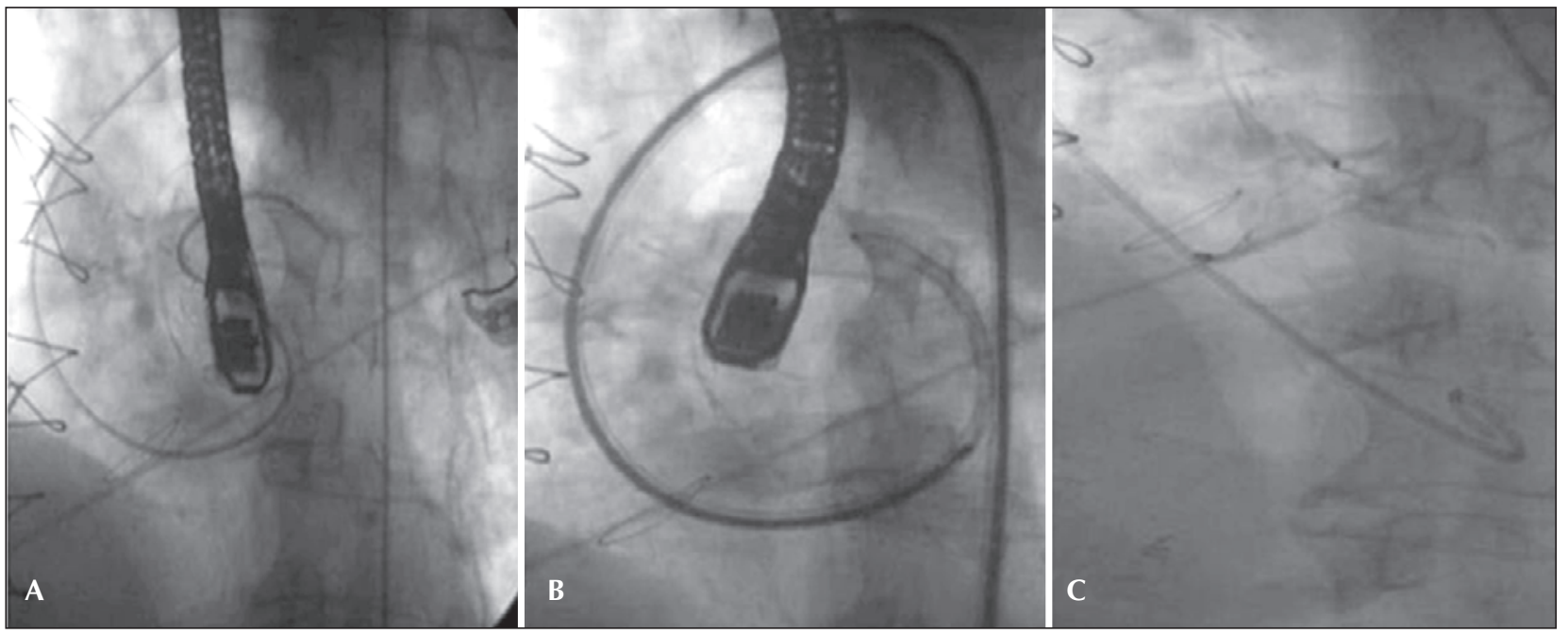

Figura 4 - Em A, cateter Multipurpose dentro do pseudoaneurisma. Em B, posicionamento da prótese Amplatzer ${ }^{\mathrm{TM}}$, por meio da bainha de Mullins. Em C, liberação da prótese.

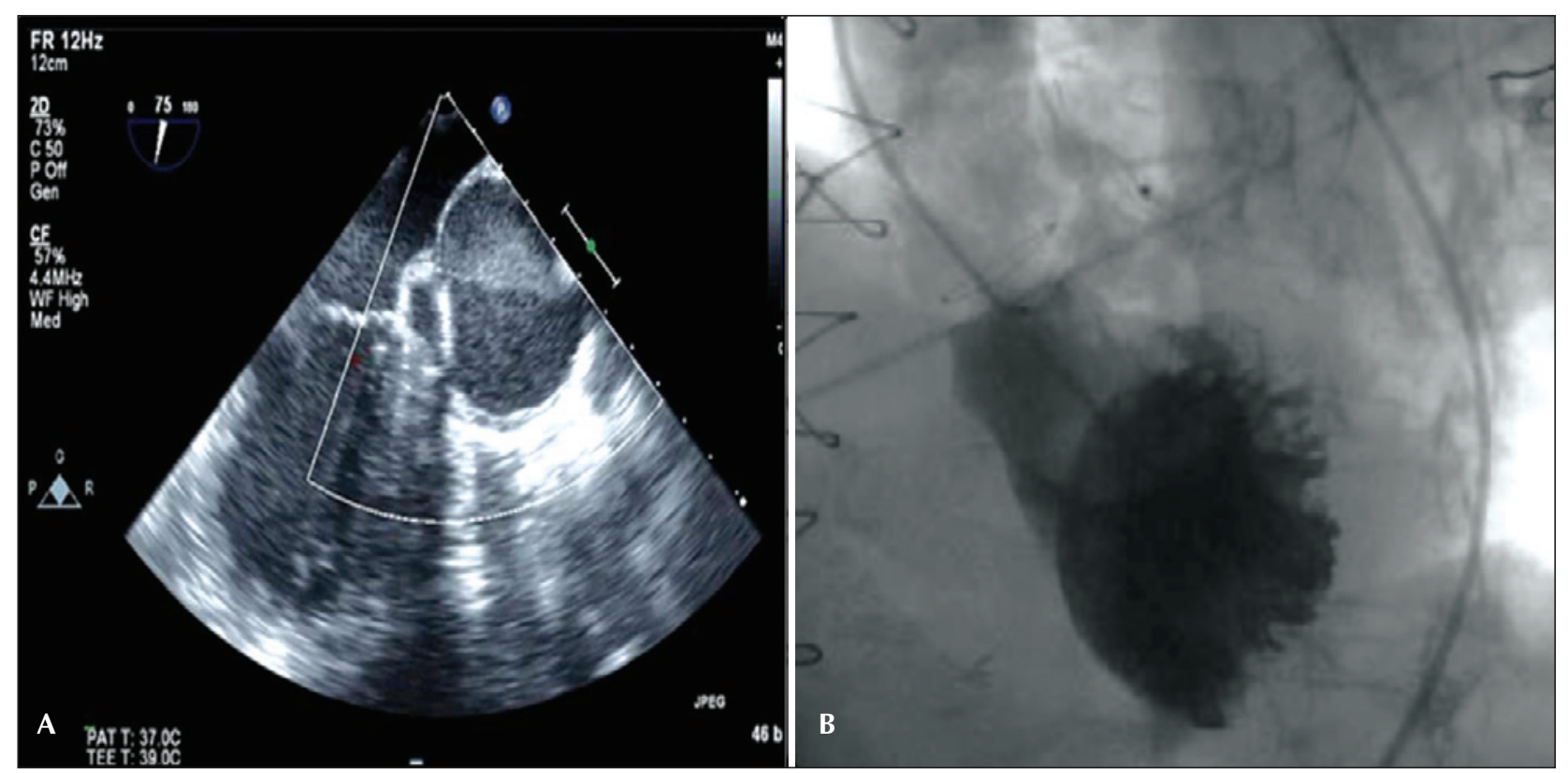

Figura 5 - Em A, ecocardiografia transesofágica, demonstrando bom posicionamento da prótese Amplatzer ${ }^{\mathrm{TM}}$ e trombose parcial do pseudoaneurisma. Em B, ventriculografia esquerda em oblíqua anterior esquerda, evidenciando ausência de fluxo dentro da cavidade do pseudoaneurisma.

do, produzindo manifestação clinica similar à insuficiência aórtica ou mitral ${ }^{10}$ ou angina, por compressão sistólica da artéria coronária esquerda. ${ }^{6,11,12}$

Para tratamento do PFMA, a correção cirúrgica é a opção mais frequentemente empregada, mesmo em pacientes assintomáticos ${ }^{3}$, com o objetivo de evitar complicações. Pode-se realizar ressecção ou reparação do defeito $^{2,12}$, com ou sem troca valvar aórtica ${ }^{5,6,12}$, ou troca da raiz aórtica por homoenxerto. ${ }^{3}$ A cirurgia, no caso relatado, acrescentaria morbidade e mortalidade significativas, pela complexidade técnica e pelo an- tecedente de duas cirurgias cardíacas. O tratamento percutâneo, já descrito em publicação prévia ${ }^{13}$, é factível e pode ser realizado de forma segura em casos selecionados, com bom resultado imediato e a médio prazo, sendo uma alternativa ao tratamento cirúrgico. Até onde se sabe, este é o segundo caso relatado na literatura de fechamento percutâneo do PFMA.

\section{CONFLITO DE INTERESSES}

Os autores declaram não haver conflito de interesses relacionado a este manuscrito. 

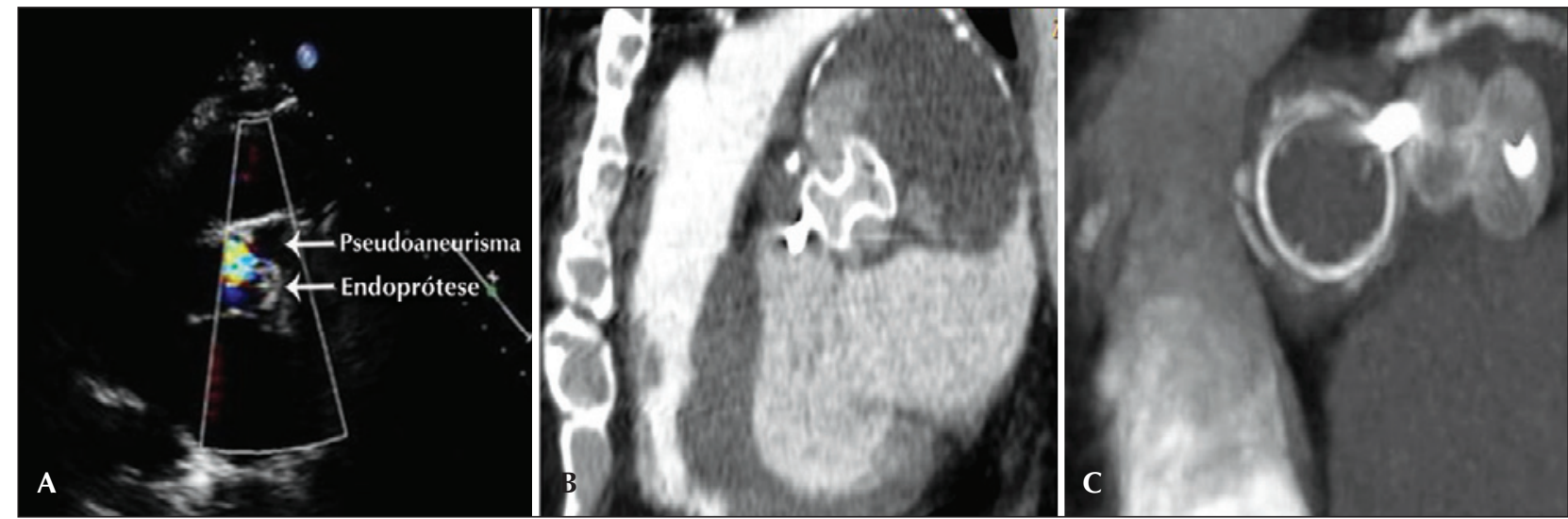

Figura 6 - Em A, ecocardiografia transtorácica, demonstrando bom posicionamento da prótese Amplatzer ${ }^{\mathrm{TM}}$, trombose parcial do pseudoaneurisma, e fluxo periprótese. Em B, tomografia, evidenciando expansão adequada da prótese e discreto fluxo contrastado periprótese. Em C, prótese Amplatzer ${ }^{\mathrm{TM}}$, sem comprometer o funcionamento da prótese valvar aórtica.

\section{REFERÊNCIAS}

1. Karalis DG, Bansal RC, Hauck AJ, Ross JJ, Applegate PM, Jutzy KR, et al. Transesophageal echocardiographic recognition of subaortic complications in aortic valve endocarditis. Circulation. 1992;86(2):353-62

2. Agirbasli M, Fadel BM. Pseudoaneurysm of the mitral-aortic intervalvular fibrosa: a long-term complication of infective endocarditis. Echocardiography. 1999;16(3):253-7.

3. Afridi I, Apostolidou MA, Saad RM, Zoghbi WA. Pseudoaneuysm of the mitral-aortic intervalvular fibrosa: dynamic characterization using transesophageal echocardiographic and Doppler techniques. J Am Coll Cardiol. 1995;25(1): 137-45.

4. Delgado C, Barturen F. Seudoaneurisma de la fibrosa mitroaórtica secundario a la desinserción parcial de una prótesis mecânica aórtica. Rev Esp Cardiol. 1999;52(5):348-50.

5. Rodrigues Borges AG, Suresh K, Mirza H, Katz JP, Simandl $\mathrm{SL}$, Bilfinger $\mathrm{T}$, et al. False aneurysm of the mitral-aortic intervalvular fibrosa after uncomplicated aortic valve replacement. J Am Soc Echocardiogr. 2002;15(7):743-5.

6. Almeida J, Pinho P, Torres JP, Garcia JM, Maciel MJ, Lima CA, et al. Pseudoaneurysm of the mitral-aortic fibrosa: myocardial ischemia secondary to left coronary compression. J Am Soc Echocardiogr. 2002;15(1):96-8.
7. Matthews RV, French WJ, Criley JM. Chest trauma and subvalvular left ventricular aneurysm. Chest. 1989;95(2):474-5.

8. Tsai IC, Fu YC, Lin PC, Lin PC, Jan SL. MDCT evaluation of congenital mitral-aortic intervalvular fibrosa aneurysm: implications for the aetiology and differential diagnosis. Pediatr Radiol. 2009;39(1):80-3.

9. Quizilbash $\mathrm{AH}$, Schwartz CJ. False aneurysm of left ventricle due to perforation of mitral-aortic intervalvular fibrosa with rupture and cardiac tamponade: rare complication of infective endocarditis. Am J Cardiol. 1973;32(1):110-3 .

10. Espinosa-Caliani JS, Montijano A, Melero JM, Montiel A. Pseudoaneurysm in the mitral-aortic intervalvular fibrosa: a cause of mitral regurgitation. Eur J Cardiothorac Surg. 2000; 17(6):757-9.

11. Antonellis J, Kostopoulos $\mathrm{K}$, Routoulas $\mathrm{T}$, Patsilinakos $\mathrm{S}$, Kranidis A, Salahas A, et al. Aneurysm of the mitral-aortic intervalvular fibrosa as a rare cause of angina pectoris. Cathet Cardiovasc Diagn. 1997;42(4):423-6.

12. Aoyagi S, Fukunaga S, Otsuka H, Akaiwa K, Yokokura $\mathrm{Y}$, Yokokura H. Left ventricular outflow tract pseudoaneurysm after aortic valve replacement: case report. J Heart Valve Dis. 2004;13(1):145-8.

13. Jiménez Valero S, García E, González Pinto A, Delcán JL. Cierre percutáneo de seudoaneurisma de la fibrosa mitroaórtica. Rev Esp Cardiol. 2005;58(12):1473-5. 